\title{
The topology at infinity of Coxeter groups and buildings
}

Michael W. Davis and John Meier

\begin{abstract}
The connectivity at infinity of a finitely generated Coxeter group $W$ is completely determined by topological properties of its nerve $L$ (a finite simplicial complex). For example, $W$ is simply connected at infinity if and only if $L$ and the subcomplexes $L-\sigma$ (where $\sigma$ ranges over all simplices in $L$ ) are simply connected. This characterization extends to locally finite buildings.
\end{abstract}

Mathematics Subject Classification (2000). 20F55, 20J05, 51E24, 57M07.

Keywords. Coxeter groups, buildings, topology at infinity, duality.

\section{Introduction}

The study of end invariants has been important in many topological applications of infinite Coxeter groups as well as buildings associated to affine Coxeter groups. For example, the first examples of compact, closed, aspherical manifolds whose universal covers are not homeomorphic to $\mathbb{R}^{n}$ were constructed using Coxeter groups that are not simply connected at infinity [9]. Borel and Serre computed the cohomology with $\mathbb{Z} G$ coefficients of $S$-arithmetic groups by studying the end topology (cohomology with compact supports) of Bruhat-Tits buildings [3]. In this paper we study the end topology of all Coxeter groups, not necessarily those associated to manifolds, as well as the end topology of all locally finite buildings. In particular we give an explicit formula for the cohomology with compact supports that holds for any locally finite building (Theorem 5.8).

Topology at infinity is essentially the study of topological properties that persistently occur in complements of compact sets. For example, a connected, locally finite $\mathrm{CW}$ complex $E$ is 1-ended if given any compact set $C \subset E$, there is a compact set $D \supset C$ such that $E-D$ is connected. The higher homology and homotopy conditions at infinity take a bit more care to state. It suffices for this paper to let $E$ be the universal cover of a finite, aspherical, $\mathrm{CW}$ complex. We say $E$ is $m$-connected at infinity if given any compact $C \subset E$ there is a compact $D \supset C$

Davis was partially supported by NSF grant DMS-9803374.

Meier thanks The Ohio State University for hosting him while on sabbatical. 
such that any map $\phi: S^{n} \rightarrow E-D$ extends to a map $\widehat{\phi}: B^{n+1} \rightarrow E-C$ for all $-1 \leq n \leq m$. Similarly, $E$ is $m$-acyclic at infinity if any $n$-cycle whose support is outside of $D$ is the boundary of an $(n+1)$-chain with support outside of $C$ for all $-1 \leq n \leq m$. In particular, since $(-1)$-acyclic means "non-empty," $(-1)$-acyclic at infinity means "non-compact" and 0-acyclic at infinity is equivalent to being 1-ended. We note that we are using integral coefficients, but one can work over any ring $R$ (with an identity element). We use the phrase simply connected at infinity to mean exactly the same thing as 1-connected at infinity, i.e., if $E$ is simply connected at infinity, it is 1-ended.

One can define homology and homotopy groups at infinity. For convenience we assume $E$ is a contractible, locally finite complex, and let $\left\{X_{n}\right\}$ be a nested, exhaustive sequence of compact subsets of $E$. This exhaustive sequence induces an inverse sequence of homology groups

$$
H_{n}\left(E-X_{0}\right) \leftarrow H_{n}\left(E-X_{1}\right) \leftarrow H_{n}\left(E-X_{2}\right) \leftarrow \cdots
$$

and the related sequence of reduced homology groups. There are also inverse sequences of homotopy groups where, in order to keep track of basepoints, one chooses a proper ray $\gamma:[0, \infty) \rightarrow E$ such that $\gamma([i, \infty)) \subset E-X_{i}$ for all $i \in \mathbb{N} \cup\{0\}$. Given such a ray, there is an induced sequence of homotopy groups

$$
\pi_{n}\left(E-X_{0}, \gamma(0)\right) \leftarrow \pi_{n}\left(E-X_{1}, \gamma(1)\right) \leftarrow \pi_{n}\left(E-X_{2}, \gamma(2)\right) \leftarrow \cdots
$$

where the morphism $\pi_{n}\left(E-X_{i}, \gamma(i)\right) \leftarrow \pi_{n}\left(E-X_{i+1}, \gamma(i+1)\right)$ is defined via inclusion and the isomorphism $\pi_{n}\left(E-X_{i}, \gamma(i)\right) \simeq \pi_{n}\left(E-X_{i}, \gamma(i+1)\right)$ induced by the path $\gamma([i, i+1])$. If $E$ is not 1-ended, then this system is affiliated with the end determined by the ray $\gamma$. Further, the inverse limit can depend on the proper homotopy class of the ray $\gamma$ (although there are no examples known where this dependence occurs in the context of an infinite, finitely presented group).

An inverse sequence of groups $\left\{\cdots \leftarrow G_{i} \leftarrow G_{i+1} \leftarrow \cdots\right\}$ is semistable (or Mittag-Leffler) if there is a function $f: \mathbb{N} \rightarrow \mathbb{N}$ such that the image of $G_{k}$ in $G_{n}$ is the same for all $k \geq f(n)$. We show that the inverse sequences of homology and homotopy groups for the spaces associated to Coxeter groups are all semistable (this is part of Lemma 3.4). This implies that "lim" ${ }^{1}$ " of these inverse systems is trivial. A further consequence is that the homotopy groups at infinity are independent of the choice of proper ray used to determine basepoints, assuming the space is 1-ended. The inverse limits do not depend on the choice of exhaustive sequence of subsets $\left\{X_{i}\right\}$. All this is to say that for the purposes of this paper we may define the end-homology and end-homotopy groups to be $H_{n}^{e}(E)=\lim _{\leftarrow}\left(H_{n}\left(E-X_{i}\right)\right)$ and $\pi_{n}^{e}(E)=\lim _{\leftarrow}\left(\pi_{n}\left(E-X_{i}\right)\right)$ (for 1-ended spaces). Although we don't take this approach here, even more can be claimed: Because these inverse sequences are semistable, information is not lost in passing to the inverse limits when thought of as topological groups (see $\S 2$ in [2]).

One can also define cohomology at infinity using the direct sequence

$$
H^{n}\left(E-X_{0}\right) \rightarrow H^{n}\left(E-X_{1}\right) \rightarrow H^{n}\left(E-X_{2}\right) \rightarrow \cdots
$$


and take $H_{e}^{n}(E)$ to be the direct limit of this sequence. (Note that $H_{e}^{n}(E)=$ $\left.H_{c}^{n+1}(E).\right)$

If a virtually torsion free group $G$ acts cellularly, properly (i.e., finite isotropy groups), and cocompactly on a locally finite, contractible CW complex $E$, then the pro-homotopy type of $\left\{E-X_{i}\right\}$ is an invariant of $G$. For example, if $E$ is simply connected at infinity, then any other such space will be simply connected at infinity, and therefore we can speak of the group being simply connected at infinity. (For background on the end topology of infinite groups, see [15] or the more succinct introduction and appendix to [16].)

In this paper we will always assume that our Coxeter groups are finitely generated. Given any finitely generated Coxeter group $W$ there is a contractible cell complex, which we are denoting $|W|$, that $W$ acts on cellularly, properly, and with finite quotient [9]. The $W$-space $|W|$ may be cellulated so that the links of vertices of $|W|$ are all isomorphic to a fixed (finite) simplicial complex $L$, where $L$ can be described purely combinatorially in terms of subsets of the generating set of $W$. The fundamental domain for the action of $W$ on $|W|$ is the cone on $L$. (Details of the construction are sketched in §3.)

We show that the topology at infinity of $|W|$ is completely determined by the "punctured topology" of the finite complex $L$. At the homological level this was done in [11] where explicit formulas for $H_{c}^{*}(|W|)$ and $H_{*}^{l f}(|W|)$ are given in terms of the cohomology and homology of $L$ relative to the subcomplexes $L-\sigma$ for $\sigma$ a simplex in $L$. We give an independent argument for such formulas in $\S 4$, where in particular we show:

$$
\widetilde{H}_{*}^{e}(|W|)=\prod_{w \in W} \widetilde{H}_{*}(L-\Omega(w))
$$

where $\Omega(w)$ is a simplex of $L$ defined in terms of the group element $w \in W$. The approach in this paper generalizes to all locally finite buildings, and we state the corresponding formulas in $\S 5$. These formulas allow us to characterize which Coxeter groups are virtual duality groups, and which buildings are duality complexes (Theorem 6.3).

In addition to extending these end-homology results to buildings, we also establish the related results on homotopy at infinity, and in Theorem 4.3 we prove the result predicted by the homology computation: The space $|W|$ is simply connected at infinity if and only if $L$, as well as each $L-\sigma$, is simply connected. In certain cases we can give fairly precise descriptions of the fundamental group at infinity. In particular, given any recursively presented group $G$, there is a finitely generated Coxeter group $W$ such that $G<\pi_{1}^{e}(W)$ (Proposition 4.5).

Combining the results on homology at infinity with the results on $\pi_{1}^{e}$, and using the pro-Hurewicz Theorem (or in our case simply applying the standard Hurewicz Theorem to complements of certain compact sets) we get the following result.

Theorem. Let $W$ be a Coxeter group, $|W|$ the usual complex on which $W$ acts, and $L$ the link of any vertex in $|W|$. Then $W$ is m-connected at infinity if and 
only if $L$ and all subcomplexes of the form $L-\sigma$ are $m$-connected.

As in the homological setting, we extend these results to locally finite buildings in $\$ 5$ where we show that the connectivity at infinity of a locally finite building is completely determined by the connectivity at infinity of its associated Coxeter group. Thus even for buildings the topology at infinity is prescribed by the punctured topology of a single finite complex.

\section{Coxeter groups}

Let $I$ be a finite index set. A Coxeter matrix on $I$ is a symmetric $I \times I$ matrix $M\left(=\left(m_{i j}\right)\right)$ with entries in $\mathbb{N} \cup\{\infty\}$ such that $m_{i j}=1$ if $i=j$ and $m_{i j} \geq 2$ if $i \neq j$. For each $i \in I$ introduce a symbol $s_{i}$ and let $S=\left\{s_{i}\right\}_{i \in I}$. The Coxeter group $W$ associated to a Coxeter matrix $M$ is the group with generating set $S$ and defining relations:

$$
\left(s_{i} s_{j}\right)^{m_{i j}}=1
$$

for all $(i, j) \in I \times I$ where $m_{i j} \neq \infty$. The natural map $S \rightarrow W$ is an injection and we identify $S$ with its image in $W$. Further, each element of $S$ has order 2 in $W$ and for all $i \neq j$ the order of the product $\left(s_{i} s_{j}\right)$ is $m_{i j}$ (for background on Coxeter groups see [4] or [7]). The pair $(W, S)$ is the Coxeter system associated to $M$.

Given a subset $J \subset I$, let $M_{J}$ denote the restriction of $M$ to $J \times J$, let $S_{J}=$ $\left\{s_{j}\right\}_{j \in J}$ and let $\left(W_{J}, S_{J}\right)$ be the Coxeter system associated to $M_{J}$. The natural map $W_{J} \rightarrow W$ takes $W_{J}$ isomorphically onto the subgroup generated by $S_{J}$.

A subset $\sigma \subset I$ is spherical if $W_{\sigma}$ is finite. (We use Greek letters to represent spherical subsets.) Let $\mathcal{S}$ denote the poset of all spherical subsets of $I$ (including the empty set). The subposet $\mathcal{S}_{>\emptyset}$ of nonempty spherical subsets of $I$ is a simplicial complex called the nerve of $(W, S)$ and is denoted $L$. The vertex set of $L$ is $I$ and a subset of $I$ spans a simplex if and only if it is spherical. Often we will ignore the distinction between a simplex and its vertex set.

Given $w \in W$, denote its word length with respect to the generating set $S$ by $l(w)$. Let $I^{*}$ denote the free monoid on $I$. Given a word $\mathbf{i}=i_{1} \cdots i_{n} \in I^{*}$, set $w(\mathbf{i})=s_{i_{1}} \cdots s_{i_{n}}$. The word $\mathbf{i}$ is $M$-reduced if $l(w(\mathbf{i}))=n$, i.e., if the word $w(\mathbf{i})$ is a minimum length representative of the group element $w(\mathbf{i})$.

For each $w \in W$ let $\Omega(w)$ be the subset of $I$ consisting of the possible letters with which an $M$-reduced word representing $w$ can end. This subset is always spherical (see [9]).

Two elements are said to be adjacent if they are connected by an edge in the Cayley graph of $(W, S)$. More explicitly, $v$ and $w$ are $i$-adjacent if $w=v s_{i}$. A gallery in $W$ is a path in the Cayley graph, so a gallery of length $n$ is a sequence $\mathbf{w}=\left(w_{0}, \ldots, w_{n}\right)$, where $w_{k}$ is adjacent to $w_{k-1}$ for $1 \leq k \leq n$. If $w_{k}$ is $i_{k}$-adjacent to $w_{k-1}$, then the gallery is of type $\mathbf{i}=i_{1} \cdots i_{n}$. Thus, $w_{n}=w_{0} w(\mathbf{i})$. The gallery is reduced if its type is $M$-reduced. (In other words, if it is a path of minimum 
length from $w_{0}$ to $w_{n}$.)

Given a subset $J \subset I$, each left coset $W_{J} w$ contains a unique element of minimum length. Let $B_{J}$ denote the set of such coset representatives. Then

$$
B_{J}=\left\{w \in W \mid l\left(s_{j} w\right)>l(w) \text { for all } j \in J\right\} .
$$

(See Ex. 3, page 37 in [4].) If $J$ is a single element, then $B_{J}$ is called a half space, and in general $B_{J}$ is called a $J$-sector. Let $\mathrm{P}_{J}: W \rightarrow B_{J}$ be the set theoretic retraction that sends $w$ to the shortest element in $W_{J} w$.

The sectors $B_{J}$ can be thought of as being based at the identity element, and in general, given a subset $J \subset I$ and an element $w \in W$, the $J$-sector at $w$ is the subset $B_{(w, J)}$ defined by $B_{(w, J)}=w B_{J}$. The retraction onto $B_{(w, J)}$ is the map $\mathrm{P}_{(w, J)}: W \rightarrow B_{(w, J)}$ defined by $\mathrm{P}_{(w, J)}=L_{w} \circ \mathrm{P}_{J} \circ L_{w^{-1}}$, where $L_{w}: W \rightarrow W$ denotes left translation by $w$.

A subset $X \subset W$ is convex if given any two elements $u, v \in X$ and a reduced gallery $\mathbf{w}=\left(w_{0}, \ldots, w_{n}\right)$ from $u$ to $v$ (i.e., $w_{0}=u$ and $w_{n}=v$ ) we have that each element $w_{k}$ of the sequence lies in $X$.

Suppose that $X$ is a subset of $W$ and that $w_{0} \in X$ is a basepoint. Then $\left(X, w_{0}\right)$ is starlike (with respect to $w_{0}$ ) if given any $x \in X$ and any reduced gallery $\mathbf{w}=\left(w_{0}, \ldots, w_{n}=x\right)$, the entire gallery lies in $X$.

Example 2.1. There are a number of convex subsets of a Coxeter group $W$. The ones below are important in the discussions that follow.

1. For any subset $J \subset I, W_{J}$ and $B_{J}$ are convex subsets of $W$.

2. If $X$ is a convex subset of $W$, then it is starlike with respect to each of its elements.

3. Suppose we order the elements of $W, W=\left\{1=w_{0}, w_{1}, w_{2}, \ldots\right\}$ in such a fashion that $l\left(w_{k+1}\right) \geq l\left(w_{k}\right)$ for all $k$. If we set $X_{n}=\left\{w_{0}, w_{1}, w_{2}, \ldots, w_{n}\right\}$, then $\left(X_{n}, 1\right)$ is starlike.

4. The previous example generalizes to sectors $B_{J}$. If we order the elements of $B_{J}, B_{J}=\left\{1=w_{0}, w_{1}, w_{2}, \ldots\right\}$, so that $l\left(w_{k+1}\right) \geq l\left(w_{k}\right)$ for all $k$, and if we define $X_{n}=\left\{w_{0}, w_{1}, w_{2}, \ldots, w_{n}\right\}$, then $\left(X_{n}, 1\right)$ is starlike.

Item (3) is critical in our arguments and was used in [9] to show that the space $|W|$ constructed in the next section is contractible.

If $\left(X, w_{0}\right)$ is starlike, then an element $x \in X-\left\{w_{0}\right\}$ is an extreme element of $\left(X, w_{0}\right)$ if no reduced gallery from $w_{0}$ to $x$ can be continued past $x$ and still remain in $X$. In other words, $x$ is an extreme element if for any reduced gallery $\mathbf{w}=\left(w_{0}, \ldots, w_{n}\right)$ in $X$ starting at $w_{0}$, if $w_{k}=x$, then $k=n$.

Given an arbitrary subset $X \subset W$ and an element $x \in W$ define the inward and outward subsets of $I$ (with respect to the pair $(X, x)$ ) by

$$
I_{\downarrow}(X, x)=\left\{i \in I \mid x s_{i} \in X\right\}
$$


and

$$
I_{\uparrow}(X, x)=\left\{i \in I \mid x s_{i} \notin X\right\} .
$$

For any pair $(X, x), I$ is the disjoint union of $I_{\uparrow}(X, x)$ and $I_{\downarrow}(X, x)$, and $I_{\uparrow}(X, x)=$ $I_{\downarrow}(\check{X}, x)$ where $\check{X}=W-X$ is the complement in $W$ of $X$.

Lemma 2.2. Suppose $\left(X, w_{0}\right)$ is a starlike subset of $W$ and that $x \in X$ is an extreme element. Then $I_{\downarrow}(X, x)=\Omega\left(w_{0}^{-1} x\right)$.

Proof. The inclusion $\Omega\left(w_{0}^{-1} x\right) \subset I_{\downarrow}\left(X, w_{0}\right)$ follows from the fact that $\left(X, w_{0}\right)$ is starlike, while the inclusion $\Omega\left(w_{0}^{-1} x\right) \supset I_{\downarrow}\left(X, w_{0}\right)$ follows because $x$ is an extreme element.

Lemma 2.3. Suppose $\left(X, w_{0}\right)$ is a starlike subset of $W$, that $x \in X$ is an extreme element, and set $\omega=\Omega\left(w_{0}^{-1} x\right)$ and $\check{X}=W-X$. Let $B=B_{(x, \omega)}$ be the $\omega$ sector at $x$ and let $\rho=\mathrm{p}_{(x, \omega)}: W \rightarrow B$ be the retraction defined above. Then $\rho(\check{X})=B-\{x\}$.

Proof. We have $\rho^{-1}(x)=x W_{\omega}$. Moreover, $x W_{\omega} \subset X$ and $B-\{x\} \subset \check{X}$, since $\left(X, w_{0}\right)$ is starlike. The lemma follows.

\section{The geometric realization $|W|$}

As in the previous section, $M$ is a Coxeter matrix on $I, W$ is the associated Coxeter group, $\mathcal{S}$ is the poset of spherical subsets of $I$ and $L$ is the nerve of $(W, S)$. We briefly recall the standard construction of the $W$-complex, which we are denoting $|W|$, and then explore the topology of the complements of starlike sets in $|W|$.

The geometric realization of the poset $\mathcal{S}$ is denoted $K$ and is called the fundamental chamber. The geometric realization of $\mathcal{S}_{>\emptyset}$ is the barycentric subdivision $L^{\prime}$ of $L$. Thus $K$ is the cone on $L^{\prime}$ (the empty set provides the cone point).

For each $i \in I$, let $K_{i}$ denote the geometric realization of $\mathcal{S}_{\geq\{i\}}$. We call $K_{i}$ the mirror of $K$ associated to $i$. For each $\sigma \in \mathcal{S}_{>\emptyset}$, let $v_{\sigma}$ denote the barycenter of $\sigma$ in $L^{\prime}$. Then $K_{i}$ can be identified with the closed star of $v_{\{i\}}$ in $L^{\prime}$.

For each nonempty subset $J \subset I$, set

$$
K^{J}=\bigcup_{j \in J} K_{j}
$$

Thus, $K^{I}=L^{\prime}$. For each $\sigma \in \mathcal{S}$, set

$$
L-\sigma=K^{I-\sigma} .
$$

In other words, $L-\sigma$ is the complement of a regular neighborhood of $\sigma$ in $L^{\prime}$. It is homotopy equivalent to $L$ with the closed simplex $\sigma$ removed and also to the full subcomplex of $L$ spanned by $I-\sigma$. 
Set

$$
W \mathcal{S}=\coprod_{\sigma \in \mathcal{S}} W / W_{\sigma} .
$$

$W \mathcal{S}$ is the poset of spherical cosets, with the partial order being given by inclusion. We denote its geometric realization by $|W|$. The inclusion $\mathcal{S} \hookrightarrow W \mathcal{S}$ defined by $\sigma \mapsto W_{\sigma}$ induces an inclusion $K \hookrightarrow|W|$ and we identify $K$ with its image in $|W|$. Similarly, the orbit projection $W \mathcal{S} \rightarrow \mathcal{S}$ defined by $w W_{\sigma} \mapsto \sigma$ induces a projection $|W| \rightarrow K$ which factors through a homeomorphism $W \backslash|W| \rightarrow K$. Thus, $K$ is a fundamental domain for the $W$-action on $|W|$ and the orbit projection $|W| \rightarrow K$ restricts to the identity on $K$. Because of this, we let $|1|$ denote the canonical image of $K$ in $|W|$ and let $|w|=w K \subset|W|$. In addition, for any $J \subset I,|w|^{J}=w K^{J}$. (The complex $|W|$ is often referred to as " $\Sigma$ " in the literature.)

For any subset $X \subset W$, let

$$
|X|=\bigcup_{x \in X}|x| .
$$

Next we prove some lemmas necessary for calculating the algebraic topology at infinity of Coxeter groups.

Lemma 3.1. For any $\sigma \in \mathcal{S}_{>\emptyset}, K$ deformation retracts onto $K^{\sigma}$.

Proof. $K^{\sigma}$ is a subcomplex of $K$ and $K^{\sigma}$ and $K$ are both contractible.

Lemma 3.2. For any subset $J \subset I$, the geometric realization $\left|B_{J}-1\right|$ deformation retracts onto $|1|^{I-J}\left(\simeq K^{I-J}\right)$.

Proof. Let $\left(X_{1}, 1\right)$ be the starlike subset defined by

$$
X_{1}=\{1\} \cup\left\{s_{i} \mid i \in I-J\right\} .
$$

Extend this, as in Example 2.1(4), to an increasing sequence of finite, starlike subsets $X_{1} \subset X_{2} \subset X_{3} \subset \cdots$ that exhaust $B_{J}$ and so that each $X_{m}-X_{m-1}=$ $\left\{x_{m}\right\}$ is a single extreme element. It follows that $\left|x_{m+1}\right| \cap\left|X_{m}\right|=\left|x_{m+1}\right|^{\omega}$ where $\omega \in \mathcal{S}$ is $\Omega\left(x_{m+1}\right)$. By Lemma $3.1,\left|X_{m+1}\right|$ deformation retracts onto $\left|X_{m}\right|$ for any $m \geq 1$, hence $\left|B_{J}\right|$ deformation retracts onto $\left|X_{1}\right|$ implying $\left|B_{J}-1\right|$ deformation retracts onto $\left|X_{1}-1\right|$, which deformation retracts onto $|1|^{I-J}$.

This argument even works when $J=\emptyset$, in which case $\left|B_{J}\right|=|W|$. The conclusion is that $|W-1|$ retracts onto $|1|^{I}(\simeq L)$.

In many of the arguments that follow we will begin by letting $\left\{X_{i}\right\}$ be an exhaustive sequence of starlike subsets of $W$, as in Example 2.1(3). We then consider the subcomplexes $|\check{X}| \subset|W|$. It should be noted that while $\check{X}=W-X$, it is not that case that $|\check{X}|=|W|-|X|$ as $|\check{X}| \cap|X| \neq \emptyset$. However, this is only a minor technical annoyance, since given any finite starlike set $X$, there is a compact subset of $|X|$ whose complement is homotopy equivalent to $|\check{X}|$. 
Lemma 3.3. Let $\left\{X_{i}\right\}$ be a nested, exhaustive sequence of finite starlike subsets of $W$. Then there is a nested, exhaustive sequence of finite compact subsets of $|W|$ such that the complements are homotopy equivalent to $\left\{\left|\check{X}_{i}\right|\right\}$.

Proof. Let $X$ be any subset of $W$ and define $i(|X|)$ to be the complement of an open regular neighborhood $|\check{X}|$, e.g., $i(|X|)$ could be the complement of the open simplicial neighborhood of $|\check{X}|$ in the barycentric subdivision of $|W|$. We call $i(|X|)$ the closed interior of $X$ and the complement of $i(|X|)$ is homotopy equivalent to $|\check{X}|$.

Lemma 3.4. Suppose that $\left(X, w_{0}\right)$ is a starlike subset of $W$, that $x$ is an extreme element of $X$, and that $Y=X-x$. Set $\omega=\Omega\left(w_{0}^{-1} x\right)$. Then

1. $|\check{Y}|$ is homotopy equivalent to $|\check{X}|$ with $|x|^{I-\omega}$ coned off. $\left(|x|^{I-\omega} \simeq L-\omega\right)$

2. $|\check{X}|$ retracts onto $|x|^{I-\omega}$.

3. $H_{*}(|\check{X}|) \simeq \widetilde{H}_{*}(|\check{Y}|) \oplus H_{*}(L-\omega)$.

Suppose further that $W$ is infinite (so that $L$ is not a simplex). Then

4. $\pi_{0}(|\check{X}|) \rightarrow \pi_{0}(|\check{Y}|)$ is surjective.

5. If we choose basepoints $\left\{p_{i}\right\}_{1 \leq i \leq k}$ in each path component of $|x|^{I-\omega}$, then the $p_{i}$ 's lie in distinct components of $|\check{X}|$ and $\pi_{1}\left(|\check{Y}|, p_{1}\right)$ is a quotient of the free product $\pi_{1}\left(|\check{X}|, p_{1}\right) * \cdots * \pi_{1}\left(|\check{X}|, p_{k}\right)$.

Proof. We have $|\check{Y}|=|\check{X}| \cup|x|$ and $|\check{X}| \cap|x|=|x|^{I-\omega} \simeq L-\omega$. Since $|x|(\simeq K)$ is contractible, statement (1) follows. As in Lemma 2.3 , let $B$ be the $\omega$-sector at $x$ and let $\rho: W \rightarrow B$ be the set theoretic retraction. Then $\rho$ induces a topological retraction, denoted by the same letter, $\rho:|W| \rightarrow|B|$ of geometric realizations. By Lemma 2.3, $\rho(|\check{X}|)=|B-x|$, and by Lemma 3.2, $|B-x|$ deformation retracts onto $|x|^{I-\omega}$. This proves (2). To prove (3), consider the exact sequence of the pair $\left(|\check{X}|,|x|^{I-\omega}\right)$. By excision, $H_{*}\left(|\check{X}|,|x|^{I-\omega}\right) \simeq H_{*}(|\check{Y}|,|x|)$. Since $|x|$ is contractible, $H_{*}(|\check{Y}|,|x|)$ is the reduced homology of $|\check{Y}|$. So the exact sequence of the pair gives

$$
H_{*}(L-\omega) \rightarrow H_{*}(|\check{X}|) \rightarrow \widetilde{H}_{*}(|\check{Y}|),
$$

and by statement (2) the first map is a split monomorphism. Hence $H_{*}(|\check{X}|) \simeq$ $\widetilde{H}_{*}(|\check{Y}|) \oplus H_{*}(L-\omega)$.

Assuming that $W$ is infinite, (4) follows from (1) and the fact that $L-\omega$ is nonempty.

To establish (5), enumerate the path components of $|\check{X}|$ as $\left\{|\check{X}|_{i}\right\}$ so that $p_{i} \in|\check{X}|_{i}$. Since $|\check{Y}|$ is homotopy equivalent to $|\check{X}|$ with a copy of $L-\omega$ coned off, it follows that

$$
L-\omega=\bigcup_{i=1}^{k}(L-\omega)_{i}
$$

for a set of disjoint subcomplexes $(L-\omega)_{i}$, where $(L-\omega)_{i} \subset|\check{X}|_{i}$. Hence $\pi_{1}(|\check{Y}|)$ is the free product of the fundamental groups of the $|\check{X}|_{i}$ with the $\pi_{1}\left((L-\omega)_{i}\right)$ killed off. 
As was mentioned in the introduction, it follows immediately from 3.4(3) that the induced inverse systems of homology groups are all semistable. Statement (5) in the above lemma can be phrased as saying "Coxeter groups are $\pi_{1}$-semistable at each end," a result first proved by Mihalik using a very different argument (Theorem 1.1 in [19]).

\section{The topology at infinity of Coxeter groups}

Formulas for $H_{*}^{l f}(|W|)$ and $H_{c}^{*}(|W|)$ are given in [11], from which one can deduce the formula given in Theorem 4.1 below. However, we can establish this formula quite quickly using the results of $\S 3$. The proof of 4.1 then applies with only minor modifications to locally finite buildings.

Theorem 4.1. Let $W$ be an infinite, finitely generated Coxeter group. Then

$$
\widetilde{H}_{*}^{e}(|W|)=\prod_{w \in W} \widetilde{H}_{*}(L-\Omega(w)) .
$$

Proof. We can exhaust the group $W$ by a sequence of finite starlike subsets $\left\{X_{i}\right\}$ where, as in Example 2.1(3), $X_{i+1}=X_{i} \cup\left\{w_{i+1}\right\}$. By Lemma 3.3 there is an associated filtration by the closed interiors of the $\left|X_{i}\right|$ such that the complements are homotopy equivalent to $\left|\check{X}_{i}\right|$. The induced inverse system of homology groups $\left\{H_{*}\left(\left|\check{X}_{m}\right|\right)\right\}$ is, by Lemma $3.4(3)$, equivalent to

$$
\cdots \leftarrow H_{*}\left(\left|\check{X}_{m}\right|\right) \leftarrow \widetilde{H}_{*}\left(\left|\check{X}_{m}\right|\right) \oplus H_{*}(L-\omega) \leftarrow \cdots
$$

with the bond being given by killing the $H_{*}(L-\omega)$ factor. By induction, the $i^{t h}$ term is $\simeq \widetilde{H}_{*}(L) \oplus \widetilde{H}_{*}\left(L-\omega_{1}\right) \oplus \cdots \oplus H_{*}\left(L-\omega_{i}\right)$, and the formula follows.

Corollary 4.2. A finitely generated Coxeter group $W$ (with associated nerve L) is m-acyclic at infinity if and only if for each $\sigma \in \mathcal{S}, L-\sigma$ is m-acyclic. In particular, $W$ is 1-ended if and only if each $L-\sigma$ is connected.

Theorem 4.3. Let $W$ be a Coxeter group with $L$ the associated nerve. Then $W$ is simply connected at infinity if and only if for each $\sigma \in \mathcal{S}, L-\sigma$ is simply connected.

Proof. $(\Leftarrow)$ Let $\{1\}=X_{0} \subset X_{1} \subset X_{2} \subset \cdots$ be an exhaustive sequence of finite, starlike subsets of $W$, as in Example 2.1(3). Filter $|W|$ by the closed interiors $i\left(\left|X_{n}\right|\right)$. By Lemma 3.4(1), the complement $\left|\check{X}_{m}\right|$ is homotopy equivalent to $\left|\check{X}_{m+1}\right|$ with a copy of $L-\omega$ coned off, for some $\omega \in \mathcal{S}$. Since $L-\omega$ is simply connected, $\pi_{1}\left(\check{X}_{m}\right) \simeq \pi_{1}\left(\check{X}_{m+1}\right)$. Thus, $\pi_{1}\left(\left|\check{X}_{m}\right|\right) \simeq \pi_{1}(|\check{1}|)$ for all $m$, but $|\check{1}|=|W-1|$ deformation retracts onto $L$ by Lemma 3.2, and $L$ is assumed to be simply connected (take $\sigma=\emptyset$ ). It follows that all of the complements $\left|\check{X}_{m}\right|$ are simply connected. 
$\Leftrightarrow$ Since $|W|$ is 1-ended, each $L-\sigma$ is path connected by Corollary 4.2. Because $|W|$ is assumed to be simply connected at infinity, and it can be filtered by finite starlike subsets, it follows from Lemma 3.4(5) that for any finite starlike subset $X \subset W,|\check{X}|$ must be simply connected. Hence, by Lemma 3.4(2), if $x$ is any extreme element, then $|x|^{I-\omega} \simeq L-\omega$ is simply connected. Since the value of $\omega$ ranges over all $\sigma \in \mathcal{S}, L-\sigma$ is simply connected for any $\sigma$.

Remark 4.4. Let $W$ be a Coxeter group with more than one end. The argument above shows that $W$ is simply connected at each end if and only if for each $\sigma \in \mathcal{S}$, the components of $L-\sigma$ are simply connected.

We can now outline the proof of the theorem stated in the introduction. One could simply appeal to the pro-Hurewicz Theorem, combining the statements of Theorem 4.3 and Corollary 4.2, to produce a proof. However, one can also do this directly using the standard Hurewicz theorem applied to complements of starlike sets. In particular, if $L$ and each $L-\sigma$ is $m$-acyclic and 1-connected, it follows by Corollary 4.2 and Theorem 4.3, that the complements of starlike subsets of $|W|$ are both $m$-acyclic and 1-connected, hence they are $m$-connected. Since $|W|$ can be exhausted by a sequence of finite, starlike subsets, it follows that $|W|$ is $m$-connected at infinity. Conversely, if $|W|$ is $m$-connected at infinity, then (as in the proof of 4.3) for any finite starlike set $(X, 1),|\check{X}|$ is $m$-connected. Taking $X=W_{\sigma}$ for $\sigma \in \mathcal{S}$ we see by Lemmas 2.3 and 3.2 that $L-\sigma$ is $m$-connected for any $\sigma \in \mathcal{S}$.

Our argument in this section leads to a reasonably clear description of the fundamental group at infinity for any 1-ended Coxeter group. In studying the topology at infinity of infinite groups one expects to find analogs of the Freudenthal-Hopf Theorem: An infinite, finitely generated group has one, two or infinitely many ends. For example, Farrell showed that if $G$ is an infinite, finitely presented group containing an element of infinite order then $H^{2}(G, \mathbb{Z} G)$ is $0, \mathbb{Z}$, or infinitely generated; if $G$ is semistable at each end then $H^{2}(G, \mathbb{Z} G)$ is always free abelian, see [16].

In their work establishing a "Freudenthal-Hopf-Farrell Theorem" for the fundamental group at infinity, Geoghegan and Mihalik point out that for infinite, finitely presented, semistable groups $G$ the plausible trichotomy $-\pi_{1}^{e}(G)$ is $0, \mathbb{Z}$, or free (in a certain topological sense) of infinite rank - doesn't hold. Their example is a right angled Coxeter group whose nerve $L$ is a homology sphere. Its fundamental group at infinity then includes $\pi_{1}(L)$ (see 4.7 in [16]). We quickly review right angled Coxeter groups, and then show in Proposition 4.5 that this situation can be made fairly generic.

Right angled Coxeter groups. A Coxeter group $W$ is right angled if each of the off diagonal entries in its Coxeter matrix $M$ is either 2 or $\infty$. If $W$ is right angled, and $\sigma \in \mathcal{S}$, then $W_{\sigma} \simeq\left(\mathbb{Z}_{2}\right)^{k}$ where $k$ is the number of vertices of $\sigma$. For 
a right angled Coxeter group one can make $|W|$ into a CAT(0) space by giving each cell the metric structure of a unit Euclidean cube. Further, the nerve $L$ is a flag complex (= simplicial complex such that any complete graph in $L^{(1)}$ is the 1 skeleton of a simplex in $L$ ). One can also run this construction backwards, namely, given a flag complex $L$ with $I=L^{(0)}$ one gets a right angled Coxeter matrix $M$ defined by

$$
m_{i j}= \begin{cases}1 & \text { if } i=j \\ 2 & \text { if }\{i, j\} \text { spans an edge } . \\ \infty & \text { otherwise }\end{cases}
$$

That this piecewise Euclidean metric on $|W|$ is CAT(0) was proved by Gromov [17]. (Showing that $|W|$ admits a piecewise Euclidean, CAT $(0)$ metric when $W$ is an arbitrary Coxeter group is more subtle and was done by Moussong [20].) Gromov showed more generally that any simply connected cubical complex is $\operatorname{CAT}(0)$ if and only if the link of each vertex is a flag complex.

Proposition 4.5. Given any recursively presented group $G$, there is a 1-ended Coxeter group $W$ such that $G<\pi_{1}^{e}(W)$.

Proof. Since any recursively presented group embeds in a finitely presented group (Higman's Embedding Theorem), we can establish the proposition by showing that given any finitely presented group $G$ we can construct a Coxeter group $W$ such that $G<\pi_{1}^{e}(W)$. To do this take $L$ to be a simplicial 4-manifold whose fundamental group is $G$. Since $L$ is a manifold we can give $L$ a sufficiently fine simplicial subdivision so that $L$ is a flag complex where the boundary of the first simplicial neighborhood of any simplex $\sigma \in L$ is a sphere or a disk. Let $W$ be the corresponding right angled Coxeter group, and filter $|W|$ as in the proof of Theorem 4.1. Since $|\check{1}|$ deformation retracts onto $L, \pi_{1}(|\check{1}|) \simeq G$. Moreover, $\pi_{1}(L-\sigma) \simeq G$ since the fundamental group of a 4-manifold is not changed by removing an open ball. It follows by van Kampen's Theorem that $\pi_{1}\left(\left|\check{X}_{m}\right|\right)$ is a free product of $m$ copies of $G$, with no amalgamation since the boundary of a simplicial neighborhood of any simplex in $L$ is a sphere or a disk.

Example 4.6. Consider in particular Thompson's group $V$, the group of dyadic homeomorphisms of the Cantor set. This group is finitely presented and contains a copy of every finite group (see [8]); hence, by Proposition 4.5 there is a Coxeter group $W$ whose fundamental group at infinity contains $V$ and therefore a copy of every finite group.

Remark 4.7. The correspondence between the topology of links in $|W|$ and the end topology of $|W|$ is rare. There are various local-to-asymptotic arguments (see [5], [6] and the references cited there) but the asymptotic-to-local direction is much harder to establish in general. Extending techniques developed in [1], we can construct $W$-complexes, $E_{W}$, that are highly connected at infinity, but 
whose vertex links are not highly connected [13]. (Other examples illustrating the difficulty in the asymptotic-to-local direction are included in [6].)

\section{Buildings}

A "building" as originally defined by Tits is a certain combinatorial object. Associated to any building there is a Coxeter matrix $M$ and a Coxeter system $(W, S)$. In the classical examples of buildings $W$ is either finite (and the building is "spherical") or $W$ is a Euclidean reflection group (and the building is "affine"). The geometric realization of a spherical or (irreducible) affine building is defined to be a certain simplicial complex, each top dimensional simplex being called a "chamber". Embedded in this geometric realization are many copies of the Coxeter complex of $(W, S)$; each copy is an "apartment," and the building can be expressed as a union of apartments. In the case of spherical buildings each apartment is a sphere and in the (irreducible) affine case each apartment is a copy of Euclidean space. (See [7] for background on the classical buildings.)

Buildings associated to more general Coxeter groups arise in the theory of Kac-Moody groups as well as in the theory of graph products of groups, and are most easily defined in terms of chamber systems (see [21]). As shown in [10], in the general setting it is more appropriate to define the geometric realization of building in such a fashion that the geometric realization of each chamber is a copy of $K$ (rather than a simplex) and so that each apartment is isomorphic to $|W|$. We recall this construction below. Because of the close connection between Coxeter groups and buildings, we can extend our results on the topology at infinity for Coxeter groups to locally finite buildings.

A chamber system over a set $I$ is a set $C$ of chambers together with a family of equivalence relations indexed by $I$. Two chambers are $i$-adjacent if they are related by the equivalence relation with index $i$. A gallery in $C$ is a finite sequence of chambers $\mathbf{c}=\left(c_{0}, \ldots, c_{n}\right)$ such that $c_{k}$ is adjacent but not equal to $c_{k-1}$. If $c_{k}$ is $i_{k}$-adjacent to $c_{k-1}$, then the type of $\mathbf{c}$ is the word $\mathbf{i}=i_{1} \cdots i_{n} \in I^{*}$. Given a subset $J \subset I$, the gallery $\mathbf{c}$ is a $J$-gallery if each $i_{k}$ is in $J$. The chamber system $C$ is connected (resp. $J$-connected) if any two elements of $C$ can be connected by a gallery (resp. a $J$-gallery). A $J$-residue of $C$ is a $J$-connected component, and $J$ is the type of the residue.

One passes from arbitrary chamber systems to buildings via Coxeter group valued distance functions. Let $M$ be a Coxeter matrix with index set $I$ and let $(W, S)$ be the associated Coxeter system. Let $C$ be a chamber system over the same index set $I$. A $W$-valued distance function on $C$ is a function $\delta: C \times C \rightarrow W$ such that if $\mathbf{i} \in I^{*}$ is an $M$-reduced word, and $c, c^{\prime} \in C$, then there exists a gallery of type $\mathbf{i}$ from $c$ to $c^{\prime}$ if and only if $\delta\left(c, c^{\prime}\right)=w(\mathbf{i})$. Such a gallery is called reduced.

Definition 5.1. A chamber system $C$ is a building (of type $M$ ) if 
1. there exists a $W$-valued distance function on $C$, and

2. for each $c \in C$ and $i \in I$ there exists at least one chamber $(\neq c)$ that is $i$-adjacent to $c$.

As in $\S 2$, let $\mathcal{S}$ denote the poset of spherical subsets of $I$. A residue is spherical if it is of type $\sigma$ for some $\sigma \in \mathcal{S}$. A building $C$ is locally finite if each of its spherical residues is finite.

An apartment in $C$ is a subset $A$ that is isometric, with respect to the $W$ valued distance function, to $W$. If $A$ is an apartment in $C$ and $c_{0} \in A$, then there is a retraction $\rho:\left(C, c_{0}\right) \rightarrow\left(A, c_{0}\right)$ defined by first mapping $\left(C, c_{0}\right)$ to $(W, 1)$ via $c \mapsto \delta\left(c_{0}, c\right)$ and then identifying $(W, 1)$ with $\left(A, c_{0}\right)$.

The definitions in $\S 2$ can be generalized in a straightforward manner. A convex subset of chambers $X \subset C$ is one that is closed under taking reduced galleries between any pair of chambers in $X$. A subset $X \subset C$ is starlike, with respect to a base chamber $c_{0} \in X$, if given $c \in X$ and any reduced gallery $\mathbf{c}=\left(c_{0}, \ldots, c_{n}=\right.$ $c)$, we have $c_{k} \in X$ for $0 \leq k \leq n$. Given that $\left(X, c_{0}\right)$ is starlike, a chamber $x \in X-\left\{c_{0}\right\}$ is an extreme chamber if no reduced gallery from $c_{0}$ to $c$ can be continued past $c$ and still remain in $X$.

Given a subset $X \subset C$ and an element $c \in C$, let $I_{\downarrow}(X, c)$ denote the set of all $i \in I$ such that there is a chamber in $X$ that is $i$-adjacent to $c$. Define $I_{\uparrow}(X, c)$ to be $I_{\downarrow}(\check{X}, c)$, that is, $I_{\uparrow}(X, c)$ consists of all $i$ such that some chamber in $\check{X}$ is $i$-adjacent to $x$. As in the Coxeter group case, for any set of chambers $X$ and any $c \in C, I=I_{\uparrow}(X, c) \cup I_{\downarrow}(X, c)$. However, it will often be the case that $I_{\uparrow}(X, c) \cap I_{\downarrow}(X, c) \neq \emptyset$, as the following example indicates.

Example 5.2. Let $C$ consist of the edges in a bipartite, uniformly trivalent tree. This is a building whose associated Coxeter group is the infinite dihedral group $D_{\infty}$; the bipartite structure indicates the two types of adjacencies. Let $X$ be the convex set consisting of the darkened edges in Figure 1, and let $c$ be the central edge. Then $I_{\uparrow}(X, c)=\{\circ, \bullet\}$ and $I_{\downarrow}(X, c)=\{\bullet\}$. The difference between the Coxeter case (where these sets are disjoint) and the buildings case occurs because buildings are usually "thick," meaning that any chamber $c$ has more than one chamber $i$-adjacent to it.

Given any two chambers $c_{0}$ and $c$ of $C$ the subset of $I$ consisting of all $i$ such that some reduced gallery from $c_{0}$ to $c$ has last adjacency type $i$ is $\Omega\left(\delta\left(c_{0}, c\right)\right)$. Using this fact we get the following analog of Lemma 2.2.

Lemma 5.3. Suppose $\left(X, c_{0}\right)$ is a starlike subset of a building $C$ and that $c \in C$ is an extreme chamber. Then

1. $I_{\downarrow}(X, c)=\Omega\left(\delta\left(c_{0}, c\right)\right)$

2. $\sigma=I-I_{\uparrow}(X, c)$ is a subset of $\Omega\left(\delta\left(c_{0}, c\right)\right)$.

In particular, $\sigma$ is a spherical subset of $I$. 


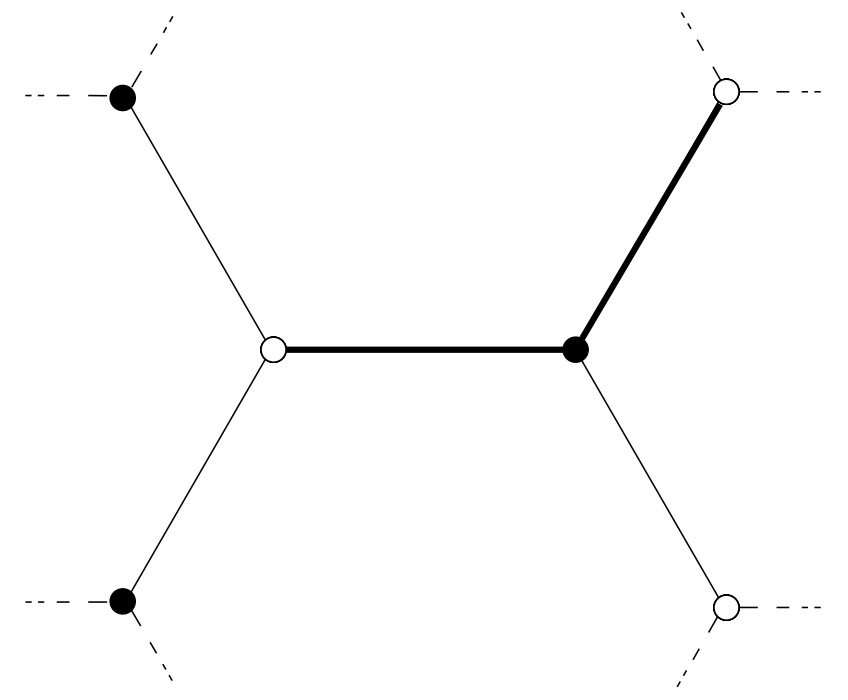

FIG. 1. The building of Example 5.2

Lemma 5.4. If $\left(X, c_{0}\right)$ is a starlike subset of $C$, then the image under the retraction to an apartment $A$ containing $c_{0},\left(\rho(X), c_{0}\right)$ is a starlike subset of $A$.

The following lemma is established in the same manner as Lemma 2.3.

Lemma 5.5. Suppose $\left(X, c_{0}\right)$ is a starlike subset of $C$, that $c \in X$ is an extreme chamber, that $\sigma=I-I_{\uparrow}(X, c)$, that $A$ is an apartment containing $c_{0}$ and $c$, and that $B \subset A$ is the $\sigma$-sector at $c$. ( $B$ is defined as in $\S$ 2.) Let $\rho_{C \rightarrow A}: C \rightarrow A$ be the retraction centered at $c$, let $\rho_{A \rightarrow B}: A \rightarrow B$ be the retraction defined in Lemma 2.3, and let $\varrho=\rho_{A \rightarrow B} \circ \rho_{C \rightarrow A}$. Then $\varrho(\check{X}) \subset B-c$ and every chamber in $B$ which is $i$-adjacent to $c$ for some $i \in I_{\uparrow}(X, c)$ is in the image.

Proof. Clearly $\varrho^{-1}(c)$ is the $\sigma$-residue containing $c$ for $\sigma=I-I_{\uparrow}(X, c)$. By definition, no element of $\check{X}$ is $i$-adjacent to $c$ for any $i \in \sigma$, and hence no element of $\check{X}$ is in $\varrho^{-1}(c)$, i.e., $\varrho(\check{X}) \subset B-c$. Also by definition, for each $i \in I_{\uparrow}(X, c)$ there is at least one $x \in \check{X}$ that is $i$-adjacent to $c$ and so $\varrho(\check{X})$ contains each chamber of $B$ that is $i$-adjacent to $c$.

Given a building $C$, let $\mathcal{C}$ be the poset of its spherical residues. By definition the geometric realization $|C|$ of $C$ is the geometric realization of the poset $\mathcal{C}$. Note that for each $c \in C,\{c\}$ is a residue of type $\emptyset$ and the map type : $\mathcal{C} \rightarrow \mathcal{S}$ restricts to an isomorphism $\mathcal{C}_{\geq\{c\}} \simeq \mathcal{S}$. It follows that the geometric realization of the subposet $\mathcal{C}_{\geq\{c\}}$ is a subcomplex of $|C|$ isomorphic to $K$. We denote this subcomplex by $|c|$ and call it a chamber. Given $i \in I$, if $\alpha$ is the $\{i\}$-residue containing $c$ then let $|c|_{i}$ 
denote the geometric realization of $\mathcal{C}_{\geq \alpha}$ (so $|c|_{i} \simeq K_{i}$ ). Similarly, for any $J \subset I$, $|c|^{J}$ is the union of the $|c|_{j}, j \in J$ (so $|c|^{J} \simeq K^{J}$ ). For any subset $X$ of $C$, put

$$
|X|=\bigcup_{c \in X}|c| .
$$

Example 5.6. The easiest (non-classical) class of examples to visualize this geometrization are the right angled buildings, i.e., those buildings whose associated Coxeter groups are right angled Coxeter groups. Locally finite right angled buildings can be constructed via graph products of finite groups. Given a finite simplicial graph $\mathcal{G}$ with groups $G_{v}$ associated to the vertices of $\mathcal{G}$, the graph product $G_{\mathcal{G}}$ is the quotient of the free product of the vertex groups modulo relations implying that $\left[G_{v}, G_{w}\right]=1$ when $\{v, w\}$ is an edge of $\mathcal{G}$.

The chambers of the building associated to $G_{\mathcal{G}}$ correspond to the elements of $G_{\mathcal{G}}$ and two chambers are $v$-adjacent if they differ by right multiplication by an element of $G_{v}$. The chambers in the geometric realization are cones on $L_{\mathcal{G}}$, where $L_{\mathcal{G}}$ is the flag complex induced by the graph $\mathcal{G}$. For example, if $\mathcal{G}$ is a 4 -cycle and each $G_{v} \simeq \mathbb{Z}_{3}$, then the building $\left|G_{\mathcal{G}}\right|$ is a $\operatorname{CAT}(0)$ square complex where each edge is a face of three squares and the link of any vertex is a copy of the utilities graph, $K_{3,3}$. (This complex is simply the product of two trivalent trees.) More generally, if $\mathcal{G}$ is an $n$-cycle for $n \geq 4$, then $\left|G_{\mathcal{G}}\right|$ is a $\operatorname{CAT}(0)$ 2-complex built out of regular $n$-gons where the links of vertices are complete bipartite graphs. (If $n \geq 5$ then $\left|G_{\mathcal{G}}\right|$ can be given a CAT $(-1)$ metric with each $n$-gon isometric to the hyperbolic right-angled $n$-gon.)

The Coxeter group $W$ associated to the building $\left|G_{\mathcal{G}}\right|$ is the graph product of $\mathbb{Z}_{2}$ 's based on the same simplicial graph $\mathcal{G}$ or, equivalently, it is the right angled Coxeter group associated to the flag complex $L_{\mathcal{G}}$. (For details on this construction see [10] and [18].)

The proof of Lemma 3.4 can be copied with only minor revisions (such as substituting Lemma 5.5 for Lemma 2.3) to give the following extension to buildings.

Lemma 5.7. Suppose $\left(X, c_{0}\right)$ is a starlike subset of a building $C$, that $c$ is an extreme chamber of $X$ and that $Y=X-c$. Set $\sigma=I-I_{\uparrow}(X, c)$. Then

1. $|\check{Y}|$ is homotopy equivalent to $|\check{X}|$ with $|c|^{I-\sigma}$ coned off. $\left(|c|^{I-\sigma} \simeq L-\sigma\right)$

2. $|\check{X}|$ retracts onto $|c|^{I-\sigma}$.

3. $H_{*}(|\check{X}|) \simeq \widetilde{H}_{*}(|\check{Y}|) \oplus H_{*}(L-\sigma)$.

If we suppose further that the associated Coxeter group $W$ is infinite, then

4. $\quad \pi_{0}(|\check{X}|) \rightarrow \pi_{0}(|\check{Y}|)$ is surjective.

5. If we choose basepoints $\left\{p_{i}\right\}_{1 \leq i \leq k}$ in each path component of $|c|^{I-\sigma}$, then the $p_{i}$ 's lie in distinct components of $|\check{X}|$ and $\pi_{1}\left(|\bar{Y}|, p_{1}\right)$ is a quotient of the free product $\pi_{1}\left(|\check{X}|, p_{1}\right) * \cdots * \pi_{1}\left(|\check{X}|, p_{k}\right)$. 
As in the Coxeter case, statements (3) and (5) of Lemma 5.7 imply that the inverse systems of homology and homotopy groups are all semistable for locally finite buildings.

Let $C$ be a locally finite building. As in the case of a Coxeter group we can order its elements $C=\left\{c_{0}, c_{1}, c_{2}, \ldots\right\}$ so that $l\left(\delta\left(c_{0}, c_{k+1}\right)\right) \geq l\left(\delta\left(c_{0}, c_{k}\right)\right)$. If $X_{m}=\left\{c_{0}, c_{1}, c_{2}, \ldots, c_{m}\right\}$, then $\left(X_{m}, c_{0}\right)$ is starlike and $c_{m}$ is an extreme chamber.

Theorem 5.8. Order the elements of a locally finite building $C$ as above. Let $\sigma\left(c_{k}\right)$ be the spherical subset defined by $\sigma\left(c_{k}\right)=I-I_{\uparrow}\left(X_{k}, c_{k}\right)$. Then

$$
\widetilde{H}_{*}^{e}(|C|) \simeq \prod_{k=0}^{\infty} \widetilde{H}_{*}\left(L-\sigma\left(c_{k}\right)\right)
$$

and

$$
\widetilde{H}_{e}^{*}(|C|)=H_{c}^{*+1}(|C|)=\bigoplus_{k=0}^{\infty} \widetilde{H}^{*}\left(L-\sigma\left(c_{k}\right)\right)
$$

Proof. As in the case of Coxeter groups one can start with the filtration by starlike subsets $\left\{X_{i}\right\}$, take the closed interiors of their geometric realizations, and then work with the complements $\left\{\left|\check{X}_{i}\right|\right\}$. An argument as in Lemma 3.2 shows that $\left|\check{X}_{0}\right|$ deformation retracts onto $\left|c_{0}\right|^{I} \simeq L$, hence $\widetilde{H}_{*}\left(\left|\check{X}_{0}\right|\right) \simeq \widetilde{H}_{*}(L)$. The homology formula follows, since Lemma 5.7(3) implies

$$
\widetilde{H}_{*}\left(\left|\check{X}_{m}\right|\right) \simeq \widetilde{H}_{*}\left(\left|\check{X}_{m-1}\right|\right) \oplus \widetilde{H}_{*}\left(L-\sigma\left(c_{m}\right)\right)
$$

and the induced morphism $\widetilde{H}_{*}\left(\left|\check{X}_{m-1}\right|\right) \leftarrow \widetilde{H}_{*}\left(\left|\check{X}_{m}\right|\right)$ is the surjection given by killing the $\widetilde{H}_{*}\left(L-\sigma\left(c_{m}\right)\right)$ factor.

The proof of the cohomology formula is similar; since we did not discuss cohomology in the Coxeter case, we include the details here. First one uses the fact that $\widetilde{H}^{*}\left(\left|\widetilde{X}_{0}\right|\right) \simeq \widetilde{H}^{*}(L)$. Then, just as in the homology case, one uses Lemma 5.7(1) to note that $\left|\check{X}_{k-1}\right|$ is homotopy equivalent to $\left|\check{X}_{k}\right|$ with a copy of $\left|c_{k}\right|^{I-\sigma\left(c_{k}\right)} \simeq$ $L-\sigma\left(c_{k}\right)$ coned off. By excision, $H^{*}\left(\left|\check{X}_{k}\right|,\left|c_{k}\right|^{I-\sigma\left(c_{k}\right)}\right) \simeq H^{*}\left(\left|\check{X}_{k-1}\right|,\left|c_{k}\right|\right)$, which is just $\widetilde{H}^{*}\left(\left|\check{X}_{k-1}\right|\right)$. The cohomology sequence of the pair $\left(\left|\check{X}_{k}\right|,\left|c_{k}\right|^{I-\sigma\left(c_{k}\right)}\right)$, along with Lemma 5.7(2), implies that

$$
\widetilde{H}^{*}\left(\left|\check{X}_{k}\right|\right) \simeq \widetilde{H}^{*}\left(\left|\check{X}_{k-1}\right|\right) \oplus \widetilde{H}^{*}\left(L-\sigma\left(c_{k}\right)\right)
$$

and the formula follows.

We note that there is a striking similarity between our formulas and the formulas found for $l^{2}$-cohomology of certain thick buildings in [14].

Remark 5.9. The main distinction between Lemmas 5.7 and 3.4 and Theorems 4.1 and 5.8 is that while $\sigma \subset \Omega\left(\delta\left(c_{0}, c\right)\right)$, in general $\sigma \neq \Omega\left(\delta\left(c_{0}, c\right)\right)$. This situation doesn't occur in the geometric realization $|W|$ of a Coxeter group $W$. As in Example 5.2, this situation will occur in any building which is not a single apartment. 
For example, if we take $c$ to be the central edge of the subset $X$ in Example 5.2, and $c_{0}$ to be the other edge of $X$, then because $I_{\uparrow}(X, c)=\{\circ, \bullet\}, \sigma(c)=\emptyset$. In particular it should be noted that while $\widetilde{H}_{*}(L)$ occurs only once in the formula for $\widetilde{H}_{*}^{e}(|W|)$, it occurs infinitely often in the formula for $\widetilde{H}_{*}^{e}(|C|)$ where $C$ is a thick building.

Remark 5.10. Notice that the formula for $\widetilde{H}_{*}^{e}(|W|)$ in Theorem 4.1 depends only on the choice of basepoint (which we chose to be 1). In Theorem 5.8, $\sigma\left(c_{k}\right)$ depends not only on the basepoint but also on the ordering. Thus, the contribution of $c_{k}$ to the formula depends on the ordering of the chambers, $\left\{c_{0}, c_{1}, c_{2}, \ldots\right\}$.

Let $C$ be a locally finite building with associated Coxeter group $W$, whose nerve is $L$. Since the formulas for $\widetilde{H}_{*}^{e}(|W|)$ and $\widetilde{H}_{*}^{e}(|C|)$ are products with factors $\widetilde{H}_{*}(L-\sigma)$, the connectivity at infinity of both $|W|$ and $|C|$ is determined by the connectivity of the complexes $L-\sigma$.

Corollary 5.11. Let $|C|$ be geometric realization of a locally finite building, with associated Coxeter group $W$ whose nerve is $L$. Then the following are equivalent:

1. $|C|$ is m-acyclic at infinity

2. $|W|$ is m-acyclic at infinity

3. $L-\sigma$ is $m$-acyclic for each $\sigma \in \mathcal{S}$.

In particular, $|C|$ is 1-ended if and only if $L-\sigma$ is connected for each $\sigma \in \mathcal{S}$.

The proof of Theorem 4.3 can be applied with only minor modifications to give

Theorem 5.12. Let $|C|$ be geometric realization of a locally finite building, with associated Coxeter group $W$ whose nerve is L. Then the following are equivalent:

1. $|C|$ is simply connected at infinity

2. $|W|$ is simply connected at infinity

3. $L-\sigma$ is simply connected for each $\sigma \in \mathcal{S}$.

Combining 5.11 and 5.12 we deduce

Theorem 5.13. Let $|C|$ be geometric realization of a locally finite building, with associated Coxeter group $W$ whose nerve is $L$. Then the following are equivalent:

1. $|C|$ is m-connected at infinity

2. $|W|$ is m-connected at infinity

3. $L-\sigma$ is m-connected for each $\sigma \in \mathcal{S}$.

One gets a concrete class of examples by considering right angled buildings corresponding to graph products of groups (Example 5.6). 
Corollary 5.14. Let $G_{\mathcal{G}}$ be a graph product of finite groups based on a finite simplicial graph $\mathcal{G}$, let $W$ be the associated right angled Coxeter group, with nerve $L_{\mathcal{G}}$. Then the graph product $G_{\mathcal{G}}$ is $m$-connected (resp. $m$-acyclic) at infinity if and only if each $L_{\mathcal{G}}-\sigma$ is m-connected (resp. $m$-acyclic) for $\sigma \in \mathcal{S}$.

\section{Punctured links, duality, and the Cohen-Macaulay property}

If $G$ is a group of type $F P$, then $G$ is an $n$-dimensional duality group if $H^{*}(G ; \mathbb{Z} G)$ is torsion free and concentrated in dimension $n$. The use of the term "duality" stems from the fact that for such groups there is a natural isomorphism between the homology and cohomology of $G$ of the form $H^{i}(G ; M) \simeq H_{n-i}\left(G ; H^{n}(G, \mathbb{Z} G) \otimes M\right)$ for all $i$ and $G$-modules $M$.

Duality properties of groups are often closely connected to the Cohen-Macaulay property for simplicial complexes. For example, in [5] it is shown that a right angled Artin group is a duality group if and only if its defining complex is CohenMacaulay. In this section we isolate our punctured link condition and consider it as a property of independent interest, and explore its connection with the CohenMacaulay property. In particular we give necessary and sufficient conditions for Coxeter groups and graph products of finite groups to be virtual duality groups in terms of punctured link conditions.

Given a simplicial complex $L$ let $\mathcal{S}(L)$ denote the poset of simplices of $L$ together with the empty set. As in $\S 3$, for each $\sigma \in \mathcal{S}(L)$, let $L-\sigma$ denote the complement of the first cellular neighborhood of $\sigma$ in the barycentric subdivision of $L$.

Definition 6.1. A finite simplicial complex $L$ has punctured homology concentrated in dimension $n$ if for each $\sigma \in \mathcal{S}(L), \widetilde{H}_{i}(L-\sigma)$ is torsion free and concentrated in dimension $n$. Equivalently, using the Universal Coefficient Theorems, one has $\widetilde{H}^{i}(L-\sigma)$ is torsion free and concentrated in dimension $n$. We write " $L$ is $P H^{n} "$ as a shorthand for this condition.

The following theorem is an immediate corollary of the cohomology formula in Theorem 5.8.

Theorem 6.2. Let $C$ be a locally finite building and let $L$ be the nerve of the associated Coxeter system $(W, S)$. Then $H_{c}^{*}(|C|)$ is torsion free and concentrated in dimension $n+1$ if and only if $L$ is $P H^{n}$.

If $G$ acts freely and cocompactly on a locally finite, contractible complex $E$, then $H^{n}(G ; \mathbb{Z} G) \simeq H_{c}^{n}(E)$. Thus one can use Theorem 6.2 to establish duality properties of groups acting on locally finite buildings.

Theorem 6.3. Let $C$ be a locally finite building with associated Coxeter group $W$, 
whose nerve is $L$, and let $G$ be a group acting freely and cocompactly on $C$. Then the following are equivalent.

1. $G$ is an $n$-dimensional duality group

2. $W$ is an $n$-dimensional virtual duality group

3. $L$ is $P H^{n-1}$.

Many of the early examples of duality groups arose from actions on affine buildings, i.e., buildings whose associated Coxeter group $W$ can be viewed as a cocompact group of reflections acting on Euclidean space $\mathbb{R}^{n}$. In this case the nerve $L$ is an $(n-1)$-sphere, which is $P H^{n-1}$. In addition to the classical cases, Theorem 6.3 also applies to graph products of finite groups (with their associated right angled buildings).

Corollary 6.4. A graph product of finite groups based on a simplicial graph $\mathcal{G}$ is a virtual duality group of dimension $n$ if and only if the induced flag complex $L_{\mathcal{G}}$ is $P H^{n-1}$.

Given a simplicial complex $L$ for each $\sigma \in \mathcal{S}(L)$ let $\operatorname{Lk}(\sigma)$ denote the link of $\sigma$. $(\operatorname{Lk}(\emptyset)=L$.

Definition 6.5. An $n$-dimensional simplicial complex $L$ is $C M_{+}$(read "CohenMacaulay plus") if for each $\sigma \in \mathcal{S}(L)$ the following two conditions hold:

1. $\widetilde{H}_{i}(\operatorname{Lk}(\sigma))=0$ for all $i \neq n-\operatorname{dim}(\sigma)-1$ and is torsion free for $i=$ $n-\operatorname{dim}(\sigma)-1$;

2. $\widetilde{H}_{i}(L) \rightarrow H_{i}(L, L-\sigma)$ is onto, for all $i$.

(By convention, $\operatorname{dim}(\emptyset)=-1$ and $\widetilde{H}_{-1}(\emptyset)=\mathbb{Z}$.)

Condition (1) is the usual definition of a Cohen-Macaulay complex. The plus condition, condition (2), is inspired by [12].

Remark 6.6. A finite tree is $P H^{0}$, and it is a 1-dimensional Cohen-Macaulay complex, but it is not $C M_{+}$. A connected graph is $C M_{+}$if and only if it is not a simplex and if it has no cut vertex or cut edge.

In [12] it is shown that if the nerve $L$ of a Coxeter group $W$ is $C M_{+}$, then $W$ is a virtual duality group. (Note: The plus condition is misstated in [12].) Lemma 6.7 below can therefore be interpreted as showing that this implication is subsumed by $(3) \Rightarrow(2)$ in Theorem 6.3 .

Lemma 6.7. If $L$ is $C M_{+}$, then it is $P H^{\operatorname{dim}(L)}$.

Proof. Let $n=\operatorname{dim}(L)$. The Cohen-Macaulay condition implies that $\widetilde{H}_{i}(L)$ is concentrated in dimension $n$; it follows from the plus condition that $\widetilde{H}_{i}(L-\sigma)$ is 
also concentrated in dimension $n$.

The next lemma essentially says that $P H^{n}$ is a hereditary condition; when the punctured homology occurs in top dimension, then the $P H^{n}$ condition is inherited by links, similar to the fact that the Cohen-Macaulay property is inherited by links.

Lemma 6.8. Let $\operatorname{dim}(L)=n$. If $L$ is $P H^{n}$, then $L k(\sigma)$ is $P H^{n-\operatorname{dim}(\sigma)-1}$ for all $\sigma \in \mathcal{S}(L)$.

Proof. It suffices to show that $P H^{n-1}$ holds for each vertex $v$. Let $\tau$ be a simplex in the link $\operatorname{Lk}(v)$ and $\sigma=\tau * v$ the corresponding simplex in $L$. (We also regard $\tau$ as a simplex in $L$.) By excision, $H_{i}(L-\tau, L-\sigma) \simeq H_{i}(C(\operatorname{Lk}(v)-\tau), \operatorname{Lk}(v)-\tau)$, so the sequence of the pair $(L-\tau, L-\sigma)$ gives:

$$
\cdots \rightarrow H_{i}(L-\sigma) \rightarrow H_{i}(L-\tau) \rightarrow H_{i}(C(\operatorname{Lk}(v)-\tau), \operatorname{Lk}(v)-\tau) \rightarrow \cdots
$$

where $C(\operatorname{Lk}(v)-\tau)$ denotes the cone on $\operatorname{Lk}(v)-\tau$. Since the homology of $L-\sigma$ and $L-\tau$ is concentrated in dimension $n$, it follows that $H_{i}(C(\operatorname{Lk}(v)-\tau), \operatorname{Lk}(v)-\tau)$ is concentrated in dimension $n$. (Note: we have used the fact that $\operatorname{dim}(L)=n$, to get that $H_{n+1}(L-\tau, L-\sigma)=0$.) Since

$$
H_{i}(C(\operatorname{Lk}(v)-\tau), \operatorname{Lk}(v)-\tau) \simeq \widetilde{H}_{i-1}(\operatorname{Lk}(v)-\tau),
$$

it follows that $H_{*}(\operatorname{Lk}(v)-\tau)$ is concentrated in dimension $n-1$. A similar computation in cohomology shows that $H^{*}(\operatorname{Lk}(v)-\tau)$ is concentrated in dimension $n-1$. It then follows from the Universal Coefficient Theorem that $H_{n-1}(\operatorname{Lk}(v)-\tau)$ is torsion free.

Corollary 6.9. Suppose $\operatorname{dim}(L)=n$. Then $L$ is $C M_{+}$if and only if it is $P H^{n}$.

In particular, a simplicial complex whose punctured homology is concentrated in top dimension is a Cohen-Macaulay complex.

\section{References}

[1] F. D. Ancel, M. W. Davis and C. R. Guilbault, CAT(0) reflection manifolds, in: Geometric Topology (Athens, GA, 1993) AMS/IP Stud. Adv. Math., volume 2, Amer. Math. Soc., Providence, RI 1997, 441-445.

[2] M. F. Atiyah and G. B. Segal, Equivariant K-theory and completion, J. Differential Geometry 3 (1969), 1-18.

[3] A. Borel and J.-P. Serre, Cohomologie d'immeubles et de groupes $S$-arithmétiques, Topology 15 (1976), 211-232.

[4] N. Bourbaki, Groupes et Algebrès de Lie, Chapters IV-VI, Masson, Paris, 1981.

[5] N. Brady and J. Meier, Connectivity at infinity for right angled Artin groups, Trans. Amer. Math. Soc. 353 (2001), no. 1, 117-132.

[6] N. Brady, J. McCammond, and J. Meier, Local-to-asymptotic topology for cocompact CAT(0) complexes, to appear, Top. Appl. 
[7] K. S. Brown, Buildings, Springer-Verlag, Berlin, Heidelberg, New York, 1989.

[8] J. W. Cannon, W. J. Floyd, and W. R. Parry, Introductory notes on Richard Thompson's groups, Enseign. Math. (2) 42 (1996), 215-256.

[9] M. W. Davis, Groups generated by reflections and aspherical manifolds not covered by Euclidean space, Ann. of Math. (2) 117 (1983), no. 2, 293-324.

[10] M. W. Davis, Buildings are CAT(0), Geometry and cohomology in group theory (Durham, 1994), 108-123, London Math. Soc. Lecture Note Ser., 252, Cambridge Univ. Press, Cambridge, 1998 .

[11] M. W. Davis, The cohomology of a Coxeter group with group ring coefficients, Duke Math. J. 91 (1998), no. 2, 297-314.

[12] M. W. Davis, Correction to "The cohomology ...", Duke Math. J. 95 (1998), 635.

[13] M. W. Davis and J. Meier, Reflection groups and CAT(0) complexes with exotic local structures, to appear, High dimensional manifold topology (Trieste, 2001), W. Lück, ed.

[14] J. Dymara and T. Januszkiewicz, Equivariant cohomology of buildings and their automorphism groups, to appear, Invent. Math.

[15] R. Geoghegan, Topological Methods in Group Theory, manuscript of a book, in progress.

[16] R. Geoghegan and M. L. Mihalik, The fundamental group at infinity, Topology 35 (1996), 655-669.

[17] M. Gromov, Hyperbolic groups, in Essays in Group Theory (ed. S.M. Gersten) M.S.R.I. Publ. 8 Springer-Verlag, Berlin, Heidelberg, New York, 1987, 75-264.

[18] J. Meier, When is the graph product of hyperbolic groups hyperbolic?, Geom. Dedicata 61 (1996), 29-41.

[19] M. L. Mihalik, Semistability of Artin and Coxeter groups, J. Pure Appl. Algebra 111 (1996), 205-211.

[20] G. Moussong, Hyperbolic Coxeter groups, PhD thesis, The Ohio State University, 1988.

[21] M. Ronan, Lectures on Buildings, Academic Press, San Diego, 1989.

Michael W. Davis

The Ohio State University

Department of Mathematics

231 W. 18th Ave

Columbus, OH 43210

USA

e-mail: mdavis@math.ohio-state.edu
John Meier

Lafayette College

Department of Mathematics

Easton, PA 18042

USA

e-mail: meierj@lafayette.edu

(Received: May 3, 2001) 